\title{
Time Savings with Once-Monthly C.E.R.A.: A Time and Motion Study Conducted in 13 Haemodialysis Centres in Italy
}

\author{
Erwin De Cock ${ }^{a}$ Persefoni Kritikou $^{b}$ Simona Raverac ${ }^{c}$ Armando Filippini $^{d}$ \\ a United BioSource Corporation, Barcelona, Spain; ${ }^{b}$ United BioSource Corporation, London, UK; ${ }^{c}$ Roche S.p.A., Monza, \\ and ${ }^{\mathrm{d}}$ Unit of Nephrology and Dialysis Policlinico, Rome, Italy
}

\section{Key Words}

C.E.R.A. · Chronic kidney disease - Time and motion studies • Haemodialysis · Dialysis · Erythropoiesis-stimulating agent · Pharmacoeconomics

\begin{abstract}
Background: We sought to document the time required by health care professionals to administer erythropoiesis-stimulating agents (ESAs) and continuous erythropoiesis receptor activator (C.E.R.A.) in the management of renal anaemia. Methods: A Time and Motion study was conducted in 13 centres in Italy. The time spent on preparation, distribution, and injection for both ESA and C.E.R.A. groups was measured. A multilevel model was run to account for the centreclustering effect. Results: The average number of ESA injections/patient/year was 89 . The average uptake of C.E.R.A. was $26 \%$. The average time per session was $1.54 \mathrm{~min}$ for ESA (95\% Cl 1.21-1.86) vs. 1.64 min for C.E.R.A. (95\% Cl 1.31-1.97). Estimated time/patient/year was $137 \mathrm{~min}$ for ESA and $20 \mathrm{~min}$ for C.E.R.A. Assuming a 100\% uptake of C.E.R.A., annual time savings/centre would be $84 \%$ (194 h). Conclusions: Substantial annual time savings on frequent anaemia managementrelated tasks were found when a switchover was made from ESAs to C.E.R.A.

(c) 2015 The Author(s)

Published by S. Karger AG, Basel
\end{abstract}

\section{Background}

The prevalence of chronic kidney disease (CKD) including end-stage renal disease (ESRD; CKD stage 5 requiring dialysis) was estimated in 2013 to be in the range between 8 and 16\% worldwide [1-3], and it is expected to increase due to an ageing population $[4,5]$. Over 2.7 million patients were reported to be treated for ESRD worldwide during 2011, of which more than 2.1 million received dialysis [6].

The disease is becoming increasingly common in Italy, and it was estimated that in 2010, in total 52,777 CKD patients were treated with dialysis, and since then, about 10,000 new patients require dialysis every year [7]. The economic impact on the Italian Health Care System was estimated at EUR 2.1 billion per year for dialysis (equivalent to approximately EUR 44,000 on a per patient basis) plus EUR 338 million indirect costs, with resource consumption increasing as the disease advances into severe stages [7]. This suggests that ESRD consumes a disproportionate share of healthcare resources.

A common complication of CKD is renal anaemia, which is manifested early on [8] and is associated with poor patient outcomes $[9,10]$. Erythropoiesis-stimulating agents (ESAs) are standard treatment for renal anaemia $[11,12]$. ESAs such as epoetin alfa, epoetin beta, and

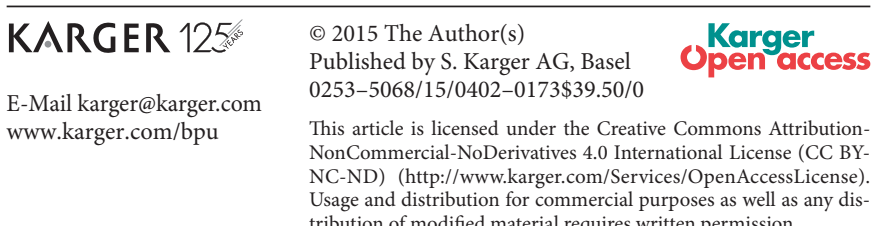

Erwin De Cock, MSc

United BioSource Corporation

Carrer Torrent del Remei 5-11, $4^{\circ}-2^{\mathrm{a}}$

ES-08023 Barcelona (Spain)

E-Mail erwin.decock@ubc.com 
epoetin zeta have relatively short half-lives and require frequent administration, ranging from 3 times a week up to once a week. Darbepoetin alfa can be administered once weekly to once every other week to maintain patients' haemoglobin $(\mathrm{Hb})$ levels within the recommended target range $[13,14]$. A recent study showed that extending its frequency to once monthly resulted in a lower $\mathrm{Hb}$ level despite a significant dose increase [15].

The frequent administration of traditional ESAs can greatly impact the workload of renal healthcare teams, as well as the patient time spent on treatment.

Mircera ${ }^{\circledR}$ (methoxy polyethylene glycol-epoetin beta), a continuous erythropoietin receptor activator (C.E.R.A.), has been proved to smoothly correct anaemia and maintain $\mathrm{Hb}$ levels within the desired target range, when administered once monthly in patients with CKD both on and not on dialysis [15-17].

Compared to traditional ESAs, C.E.R.A. has been shown to require substantially less health care personnel (HCP) time associated with routine anaemia-management tasks [18-20]. Those studies were performed in 6 centres or less per country, therefore limiting the ability to generalise results for the respective countries.

The primary objective of this time and motion (T\&M) study was to quantify the HCP time associated with routine anaemia management tasks when using traditional ESAs and C.E.R.A. in patients with ESRD on haemodialysis (HD) in a large sample of HD centres in Italy.

The secondary objective was to model potential time savings for the HCPs associated with the adoption of C.E.R.A.

\section{Methods}

\section{Study Design}

This T\&M was a prospective, non-interventional, observational study, conducted in $13 \mathrm{HD}$ centres in Italy (8 private and 5 public centres), treating ESRD patients having anaemia with ESAs (including C.E.R.A.). Within this paper, the group of non-C.E.R.A. drugs (shorter-acting ESAs) is defined as 'Other ESAs'.

The data collected were not linked to any patient demographics, and informed consent was not required. However, formal ethics approval was obtained in all public centres (no ethics approval was required at the 8 private centres).

\section{Study Procedures and Data Collection}

T\&M methodology consists of decomposing a process (here anaemia management) into its constituting tasks and observing and recording time taken by HCPs to perform selected tasks repeatedly [21].

T\&M data were collected during HD sessions in which one or more patients received 'Other ESAs' and/or C.E.R.A.
Selected tasks were defined by a clear and unambiguous start and stop point such that they were not intertwined with other HDrelated activities. Tasks that were not expected to be different by type of ESA drug (such as blood sampling, physician consultation, administrative duties) were not included in the study. Case Report Forms, one for 'Other ESAs' and one for C.E.R.A., were tailored to each centre's practice after obtaining information during an interview with a centre staff member. The interview focused on anaemia management practices, chronological order of activities, and descriptions of tasks.

Three tasks were retained: preparation, distribution, and injection of ESAs. Preparation included checking the type of ESA that the patient needed, collecting the drug from the fridge, and putting the syringe ready for distribution. Distribution covered physical distribution of syringes to the patient HD chairs. Administration included checking patient identification and correct ESA drug and dosing, and administration of ESA subcutaneously or via a dialysis line. Preparation and distribution could be performed per group of patients, and every individual patient was injected. For 'per group' tasks, a target sample size of 20 observations was set for 'Other ESAs' and 15-20 for C.E.R.A. For 'per patient' tasks, the sample size was 40 observations for 'Other ESAs' and 30-40 observations for C.E.R.A. (target sample sizes for C.E.R.A. were lower due to once-monthly administration schedule).

Time measurements were performed by trained observers using a stopwatch. Data clarification process was performed through data clarification forms issued to the centres. Time values could be adjusted if an 'unexpected event' was reported that affected the active time measured.

\section{Statistical Analyses}

This was a descriptive study of real-world observations. No sample size calculations were performed, as no a priori statistical differences in HCP time per single administration between 'Other ESAs' and C.E.R.A. were expected.

The unit of analysis was 'time per task', where each task constituted an independent sample, assuming no interaction between task times per patient.

For 'per group' tasks, time data were adjusted to 'time per patient'. As the number of patients for group tasks (preparation and/or distribution, depending on the centre) was typically larger for 'Other ESAs' than for C.E.R.A. sessions, a random intercept generalised linear mixed effects model assuming gamma distribution with a log-link function (hereafter referred to as 'multilevel model') was used in order to adjust for potential differences in time based on the size of the group.

To generate country results, data from all centres were pooled. For each task, a multilevel model was fitted to account for the potential clustering of centre data (for 'per group' tasks, a group size of $\mathrm{n}=4$ patients for C.E.R.A. was assumed, to reflect the mean group size for 'Other ESAs'). The total time per patient was calculated by summing the time estimates of all 3 tasks and 95\% CIs for the composite were derived using the variance sum law I.

Analysis was performed using Statistical Analysis Software version 9.1 (SAS Institute Inc., Cary, N.C., USA).

Modelling the Impact of Once-Monthly C.E.R.A.

For each centre, an average administration frequency of 'Other ESAs' injections (defined as 'number of sessions per patient per year') was calculated based on the distribution of ESA products used and 
Table 1. Characteristics of ESA administration per centre

\begin{tabular}{|c|c|c|c|c|c|c|c|c|c|c|c|c|c|c|}
\hline & $\mathrm{C} 1$ & $\mathrm{C} 2$ & C3 & $\mathrm{C} 4$ & $\mathrm{C} 5$ & C6 & $\mathrm{C} 7$ & $\mathrm{C} 8$ & C9 & $\mathrm{C} 10$ & $\mathrm{C} 11$ & $\mathrm{C} 12$ & C13 & Average \\
\hline $\begin{array}{l}\text { Number of ESRD patients } \\
\text { receiving ESA (including C.E.R.A.) }\end{array}$ & 80 & 83 & 87 & 65 & 81 & 70 & 83 & 170 & 86 & 36 & 56 & 82 & 76 & 81 \\
\hline \% of C.E.R.A. uptake & 25 & 11 & 28 & 29 & 35 & 29 & 17 & 10 & 24 & 36 & 20 & 41 & 37 & 26 \\
\hline $\begin{array}{l}\text { Average number of 'Other ESA' } \\
\text { injections per patient per month } \\
\text { (excluding patients receiving } \\
\text { C.E.R.A.) }\end{array}$ & 5.6 & 9.3 & 7.2 & 5.8 & 7.9 & 7.6 & 3.7 & 11.1 & 7.9 & 8.7 & 2.8 & 12.5 & 6.6 & 7.4 \\
\hline $\begin{array}{l}\text { Estimated number of 'Other ESA' } \\
\text { injections per patient per year } \\
\text { (excluding patients receiving } \\
\text { C.E.R.A.) }\end{array}$ & 67 & 111 & 86 & 69 & 94 & 91 & 45 & 133 & 94 & 104 & 33 & 150 & 79 & 89 \\
\hline
\end{tabular}

* Based on data from centres at time of interview.

injection frequency. For C.E.R.A., all centres confirmed a oncemonthly schedule. This was used to estimate the annual time for anaemia management (per patient and per centre, using centre size at the time of study set-up) as well as to estimate the time savings comparing scenarios of 100\% 'Other ESAs' and 100\% C.E.R.A. adoption.

\section{Scenario Analysis}

A scenario analysis was performed to estimate the annual time required for the administration of short-acting ESAs (epoetin alfa, epoeting beta, and epoeting zeta) vs. medium-acting ESAs (darbepoetin alfa) and its impact on potential time savings with a $100 \%$ adoption of C.E.R.A. Administration frequencies for short-acting and medium-acting ESAs were assumed to be 3 times per week and one time per week, respectively, and were applied to the average total time per session for 'Other ESAs' (as described earlier) to calculate the estimated annual time per patient by ESA category. Results were extrapolated at the centre level, assuming that all patients received short-acting ESAs vs. medium-acting ESAs, and compared to $100 \%$ uptake of C.E.R.A.

\section{Results}

\section{Characteristic of HD Centres}

The average number of ESRD patients receiving ESA treatment was 81 (range 36-170; table 1).

Participating centres had up to 5 ESAs available on their formularies for the treatment of renal anemia: epoetin alfa $\left(\right.$ binocrit ${ }^{\circledR}$, and eprex ${ }^{\circledR}$ ), epoetin beta (neorecormon ${ }^{\circledR}$ ), epoetin zeta $\left(\right.$ retacrit $\left.^{\circledR}\right)$, darbepoetin alfa $\left(\operatorname{aranesp}^{\circledR}\right.$ ) and C.E.R.A. The average level of C.E.R.A. uptake at the time of study set-up was 26\% (range 11-41), mainly through the intravenous route (table 1). Patients receiving 'Other ESAs' were distributed as follows: $54 \%$ epoetin alfa, $2 \%$ epoetin beta, $8 \%$ epoetin zeta, and $36 \%$ darbepoetin alfa. Of the 'Other ESAs', 97\% received drug intravenously. The distribution of patients based on the type of 'Other ESAs' received is presented in figure 1.

The average number of 'Other ESA' injections per patient per month was 7.4 (range: $2.8-12.5$ ), or 89 injections per year compared to 12 for C.E.R.A., resulting in an average 77 ' Other ESA' injections avoided following a switch to C.E.R.A. (range: 21-138; table 1).

\section{Observed Time per Patient per Session for 'Other}

ESAs'vs. C.E.R.A.

When pooling data from all centres, a centre effect was detected for all tasks (while on a centre-level basis, with the exception of 2 centres, group size was always found to be a predictor of time). The average total time per session was $1.54 \mathrm{~min}$ (92 s) for 'Other ESAs' (95\% CI 1.21-1.86 $\mathrm{min}$; or $73-112 \mathrm{~s}$ ) and $1.64 \mathrm{~min}$ (98 s) for C.E.R.A. (95\% CI 1.31-1.97 min; or 79-118 s). Injection administration time was similar across both groups ( $0.68 \mathrm{~min}$; or $41 \mathrm{~s}$ for 'Other ESAs' and $0.81 \mathrm{~min}$; or 49 s for C.E.R.A.), accounting for $44 \%$ and $49 \%$ of total observed time, respectively.

\section{Estimated Time per Patient per Year}

Average time per session yielded an estimated annual time per patient of $137 \mathrm{~min}$ for 'Other ESAs' (range 65277) and of $20 \mathrm{~min}$ for C.E.R.A. (range 5-51), resulting in 


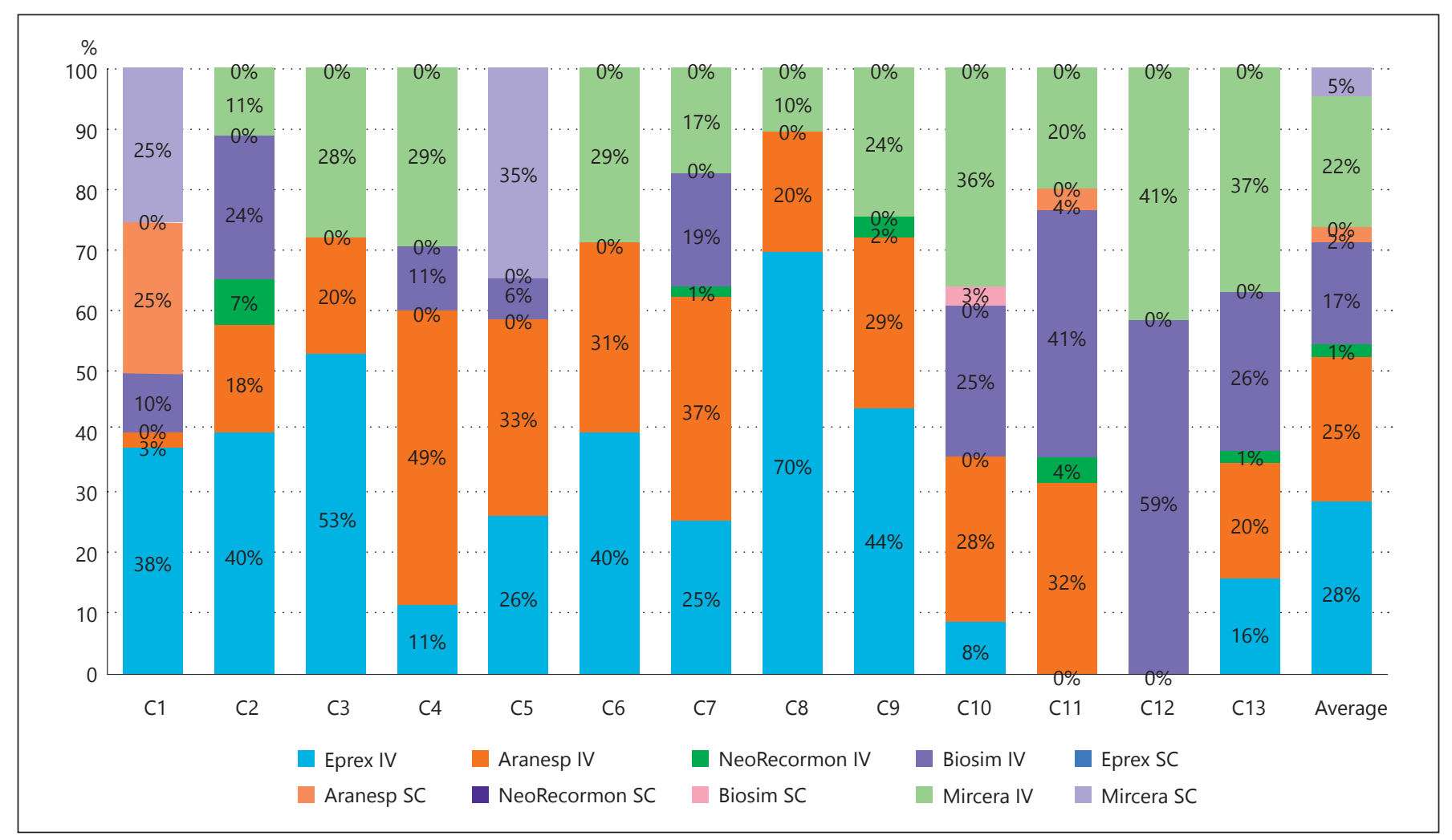

Fig. 1. Distribution of patients by type of 'Other ESAs' received.

an estimated annual time savings per patient per year of 117 min (range 49-232), or an $84 \%$ reduction (range $62-$ 95; fig. 2).

\section{Estimated Time per Centre per Year}

When extrapolating to each centre's ESRD population receiving ESAs, the annual time ranged from 80 to $719 \mathrm{~h}$ when all patients would receive 'Other ESAs' and from 8 to $74 \mathrm{~h}$ when all patients would receive C.E.R.A. (fig. 3). For 'Other ESAs', the wide range in absolute time reflects the mix of ESA products used, their respective injection frequencies, and the ESRD population in the centre. Annual time savings ranged between 50 and $658 \mathrm{~h}$ in case all patients were treated with C.E.R.A. (194 h for an average population of 81 patients, or a reduction of $84 \%$ ).

\section{Scenario Analysis}

The estimated annual time per patient was $240 \mathrm{~min}$ for short-acting ESAs, 80 min for medium-acting ESAs, and $20 \mathrm{~min}$ for C.E.R.A. For an average population of $81 \mathrm{pa}-$ tients, assuming scenarios of $100 \%$ use of short-acting ESAs, $100 \%$ use of medium-acting ESAs, or $100 \%$ use of C.E.R.A., the estimated annual time per centre was 325,
108 , and 30 h, respectively. With $100 \%$ uptake of C.E.R.A., the total time savings would be 295 and 78 h, respectively (or a reduction of $91 \%$ and $72 \%$ ).

\section{Discussion}

This T\&M study conducted among a sample of 13 Italian HD centres that provided treatment for ESRD patients generated more precise time estimates across a mix of private and public dialysis centres than those previously published for 5 private Italian centres only [18]. The study revealed that even though the active time spent on frequent anaemia management tasks (i.e. preparation, distribution, and injection of ESA drug) is small (average $1.54 \mathrm{~min}$; or $92 \mathrm{~s}$ for 'Other ESAs' and $1.64 \mathrm{~min}$; or $98 \mathrm{~s}$ for C.E.R.A.), time becomes substantial when extrapolating to an average 89 annual sessions for 'Other ESAs' vs. 12 sessions for C.E.R.A., resulting in average annual time savings of $117 \mathrm{~min}(-86 \%)$.

The substantial simplification of the anaemia management process with C.E.R.A., as measured by the relative reduction in active HCP time, can be considered an advance in general hemodialysis management. Indeed, the 


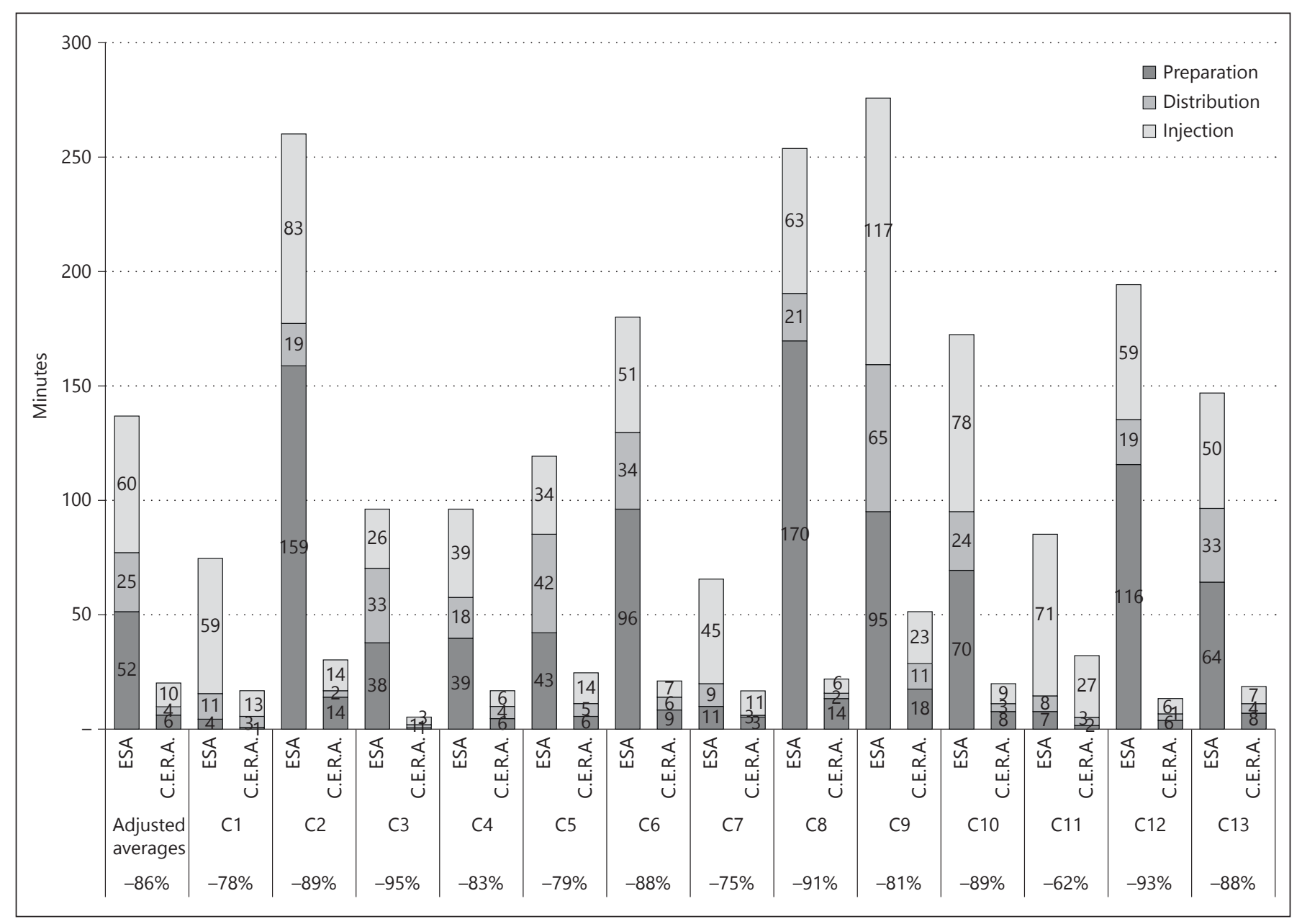

Fig. 2. Estimated time per patient per year for 'Other ESAs' vs. C.E.R.A.

time savings generated by converting to C.E.R.A. mean that dialysis centres are able to spend more time on clinical assessment and disease management, and therefore to improve overall patient care. In the short-term, it could even be argued that redistribution of staffing resources may result in financial gain through reduced overtime depending on the resource demands and funding structure.

The additional time available to help patients achieve guideline targets for key clinical parameters such as maintenance of $\mathrm{Hb}$ levels within the recommended target range may exert beneficial effects also on morbidity and mortality in patients with CKD. These potential benefits coincide with the views expressed by healthcare staff in this study who considered that time being freed up could be spent on documenting patient parameters and instructing or reinstructing patients on dialysis access care and diet.

Our findings confirm those of a previous multi-country study that included 5 centres in Italy. That study re- ported an average 102 'Other ESA' administrations per patient per year, resulting in 90 administrations avoided when switching over to C.E.R.A. (compared to 89 'Other ESA' administrations and 77 ESA administrations avoided in this study). The average total time per session was somewhat higher: $2.52 \mathrm{~min}$ (151 s) for 'Other ESAs' and $2.29 \mathrm{~min}(137 \mathrm{~s}$ ) for C.E.R.A. (compared to $1.54 \mathrm{~min}$; or $92 \mathrm{~s}$ and $1.64 \mathrm{~min}$; or $98 \mathrm{~s}$ here). The estimated annual time savings with a switch to C.E.R.A. was $93 \%$, comparable to $95 \%$ reduction estimated in the present analysis.

However, some limitations of this T\&M study should be noted.

First, variability in time for each pre-specified task was observed between centres. For example, time for injection ranged from $0.30 \mathrm{~min}(18 \mathrm{~s})$ to $2.11 \mathrm{~min}(127 \mathrm{~s})$ for 'Other ESAs' and from $0.27 \mathrm{~min}(16 \mathrm{~s})$ to $2.25 \mathrm{~min}(135 \mathrm{~s})$ for C.E.R.A., exemplifying differences in local centre practices. For this reason, the multilevel model to detect and 


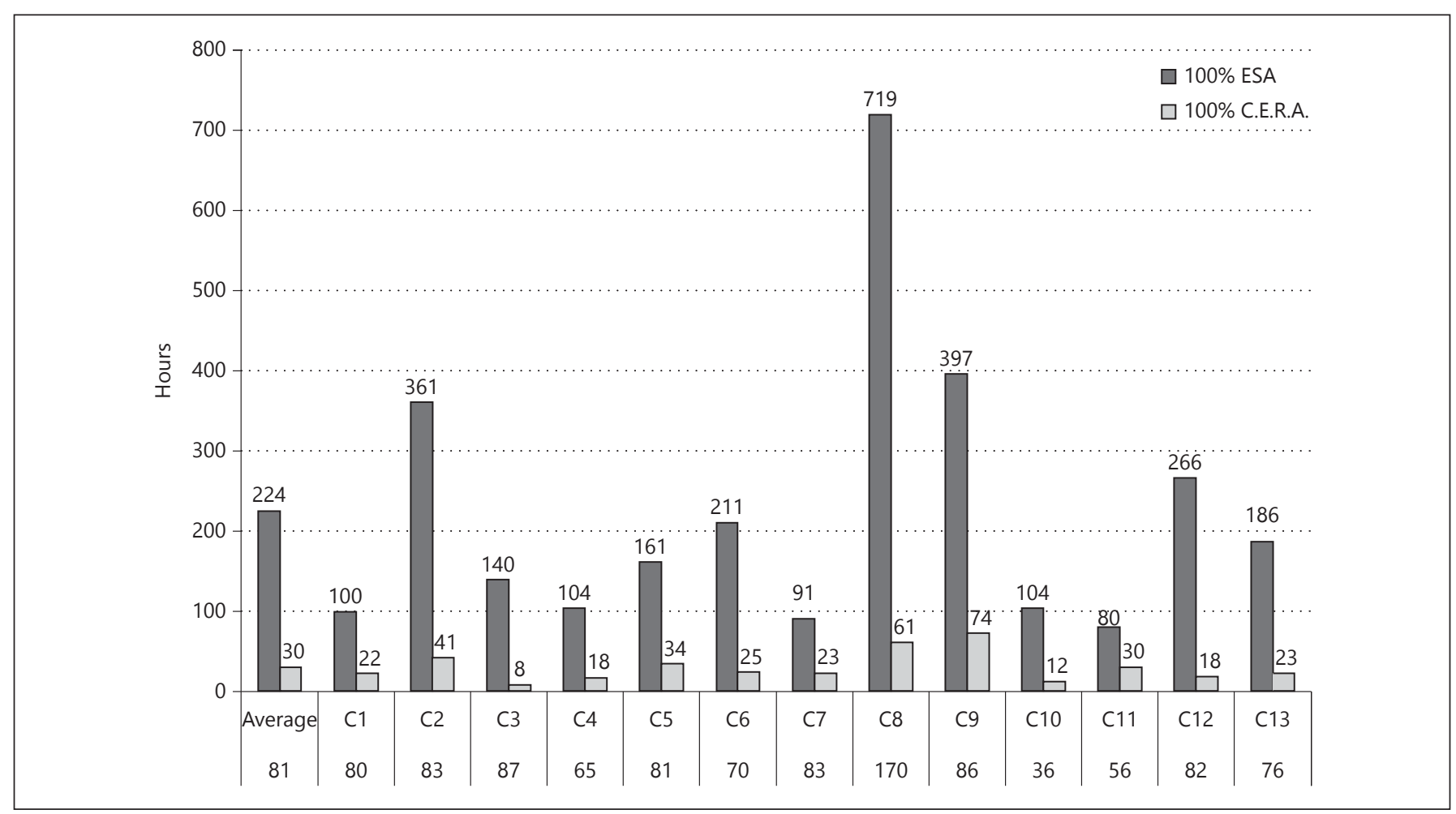

Fig. 3. Estimated time per centre per year with 100\% 'Other ESAs' vs. 100\% C.E.R.A.

correct for centre clustering of data was the most suitable method. Differences were also observed when comparing injection time for C.E.R.A. and 'Other ESAs' by centre, ranging between factor 0.87 and 3.28 (C.E.R.A. time divided by 'Other ESAs' time). Although this study did not aim at testing a hypothesis of a difference in time between both groups (such a difference is not expected), these results together with non-overlapping confidence intervals suggest true differences between both injection processes in some centres.

We aimed at minimizing within- and between-centre variability in time measurements by clearly defining the concept of active HCP time, and by developing clear and unambiguous task descriptions. As part of this process, generic task descriptions were tailored to each centre's practice, if needed. Variability in task time could also be due to potential inter-observer variability in stopwatch measurement. To mitigate this, observers in all centres received a standardised training.

Second, inevitable differences in the mix of ESA products and their administration frequencies lead to variability in the number of annual 'Other ESA' administrations per patient ranging from 33 (Centre 11) to 150 (Centre 12), impacting the total annual time and the level of savings that could be achieved with $100 \%$ adoption of C.E.R.A. (range 62-95). Note that Centre 12 had the highest proportion of patients who already switched to C.E.R.A. (41\%), which may indicate that the patients that would benefit from a less frequent dosing schedule already made the switch. Also, the comparison between scenarios of $100 \%$ of patients receiving 'Other ESAs' vs. $100 \%$ receiving C.E.R.A. assumes that drug frequency distribution observed for patients receiving 'Other ESAs' is applicable to all patients, also those who had already switched to C.E.R.A. In other words, if those patients who had switched to C.E.R.A. were previously receiving another ESA at a lower frequency, estimated annual time savings may be overestimated.

Third, estimated time per centre per year reflects the total number of patients receiving ESA at each centre at a given point in time (when the initiation interview was performed) and prevents meaningful comparison between centres. Therefore, the estimated annual time was calculated assuming that all patients were treated either with 'Other ESAs' or C.E.R.A. In the real world, staff time dedicated to all patients for the tasks being measured will lie somewhere in between, depending on the proportion 
of patients that switched to C.E.R.A. The result therefore cannot be generalised to an average Italian centre (which is not the aim of this study), but gives an indication of the annual resource requirements for both scenarios in terms of staffing for frequent anaemia management activities, and the potential for freeing up staff resources.

Lastly, it should be noted that in Italy, the prices for C.E.R.A. and 'Other ESAs' are defined at a regional level and therefore can be different from one region to another. For this reason, to explore the overall impact of a switch from 'Other ESAs' to C.E.R.A. on direct costs, a formal cost-minimisation analysis that includes drug cost and the monetary value of health care staff time should be implemented at a regional level. This was outside the scope of this study, which focused on process simplification and associated staff resource implications.

In conclusion, this study showed that both public and private HD centres in Italy spend a substantial amount of time per year on tasks related to anaemia management of ESRD patients, especially those tasks required with every ESA administration. Switching to once-monthly C.E.R.A. would allow scarce healthcare resources to be reallocated to focus on other important aspects of patient care, thereby enhancing the overall quality of dialysis care.

\section{Acknowledgments}

The authors acknowledge the financial support of this study from Roche S.p.A.

\section{Disclosure Statement}

E.D.C., P.K. and A.F.: sponsored by Roche to conduct this study. S.R.: employee of Roche.

\section{References}

1 Jha V, Garcia-Garcia G, Iseki K, Li Z, Naicker S, Plattner B, Saran R, Wang AY, Yang CW: Chronic kidney disease: global dimension and perspectives. Lancet 2013;382:260-272.

-2 De Nicola L, Donfrancesco C, Minutolo R, Lo Noce C, De Curtis A, Palmieri L, Iacoviello L, Conte G, Chiodini P, Sorrentino F, Coppo R, Vanuzzo D, Scherillo M, Giampaoli S: [Epidemiology of chronic kidney disease in Italy: current state and contribution of the CARHES study]. G Ital Nefrol 2011;28:401-407.

- 3 Garofalo C, Liberti ME, Sagliocca A, Michini C, Palmisano R, Pirro L, Provenzano M, Minutolo R, De Nicola L, Conte G: [Epidemiology and prognosis of chronic kidney disease in Italy]. G Ital Nefrol 2012;29:S3-S11.

4 Zhang QL, Rothenbacher D: Prevalence of chronic kidney disease in population-based studies: systematic review. BMC Public Health 2008;8:117.

5 Zoccali C, Kramer A, Jager KJ: Epidemiology of CKD in Europe: an uncertain scenario. Nephrol Dial Transplant 2010;25:17311733.

6 Fresenius Medical Care: ESRD Patients in 2011: A Global Perspective. http://www. vision-fmc.com/files/download/ESRD/ ESRD_Patients_in_2011.pdf (accessed June 26, 2014).

7 Cicchetti A, Ruggeri M, Codella P, Ridolfi A: I costi socio-sanitari dell'insufficienza renale cronica. Farmeconomia e Percorsi Terapeutici 2011;12:75-82.

8 Locatelli F, Del Vecchio L, Pozzoni P: Treating anemia at different stages of renal disease. J Nephrol 2007;20(suppl 12):S33-S38. $\checkmark$ Hörl WH: Anaemia management and mortality risk in chronic kidney disease. Nat Rev Nephrol 2013;9:291-301.

10 Nugent RA, Fathima SF, Feigl AB, Chyung D: The burden of chronic kidney disease on developing nations: a 21 st century challenge in global health. Nephron Clin Pract 2011; 118:c269-c277.

11 KDOQI: KDOQI clinical practice guideline and clinical practice recommendations for anemia in chronic kidney disease: 2007 update of hemoglobin target. Am J Kidney Dis 2007;50:471-530.

12 Vankar SG, Dutta P, Kohli HS, Bhansali A: Efficacy \& safety of continuous erythropoietin receptor activator (CERA) in treating renal anaemia in diabetic patients with chronic kidney disease not on dialysis. Indian J Med Res 2014;139:112-116.

13 Locatelli F, Aljama P, Bárány P, Canaud B, Carrera F, Eckardt KU, Hörl WH, Macdougal IC, Macleod A, Wiecek A, Cameron S: Revised European best practice guidelines for the management of anaemia in patients with chronic renal failure. Nephrol Dial Transplant 2004;19(suppl 2):ii1-ii47.

14 Carrera F, Disney A, Molina M: Extended dosing intervals with erythropoiesis-stimulating agents in chronic kidney disease: a review of clinical data. Nephrol Dial Transplant 2007;22(suppl 4):iv19-iv30.

15 Carrera F, Lok CE, de Francisco A, Locatelli F, Mann JF, Canaud B, Kerr PG, Macdougall IC, Besarab A, Villa G, Kazes I, Van Vlem B, Jolly S, Beyer U, Dougherty FC; PATRONUS Investigators: Maintenance treatment of renal anaemia in haemodialysis patients with me- thoxy polyethylene glycol-epoetin beta versus darbepoetin alfa administered monthly: a randomized comparative trial. Nephrol Dial Transplant 2010;25:4009-4017.

16 Minutolo R, Conte G, Cozzolino M, Polito P, Manno C, Di lorio BR, Santoro D, Di Luca M, Nappi F, Feriozzi S, Sasso FC, De Nicola L: Conversion from epoetin and darbepoetin to C.E.R.A. in non-dialysis CKD patients: a multicenter Italian prospective study in nephrology practice. Blood Purif 2013;36:69-77.

17 Mann JF, de Francisco A, Nassar G, Canaud $\mathrm{B}$ : Fewer dose changes with once-monthly C.E.R.A. in patients with chronic kidney disease. Clin Nephrol 2011;76:9-15.

18 De Cock E, Dellanna F, Khellaf K, Klatko W, Maduell F, Raluy-Callado M, Villa G: Time savings associated with C.E.R.A. once monthly: a time-and-motion study in hemodialysis centers in five European countries. J Med Econ 2013;16:648-656.

19 Saueressig U, Kwan JT, De Cock E, Sapède C: Healthcare resource utilization for anemia management: current practice with erythropoiesis-stimulating agents and the impact of converting to once-monthly C.E.R.A. Blood Purif 2008;26:537-546.

20 Schiller B, Doss S, De Cock E, Del Aguila MA, Nissenson AR: Costs of managing anemia with erythropoiesis-stimulating agents during hemodialysis: a time and motion study. Hemodial Int 2008;12:441-449.

21 Wirth P, Kahn L, Perkoff GT: Comparability of two methods of time and motion study used in a clinical setting: work sampling and continuous observation. Med Care 1977;15: 953-960. 